\title{
Verbessert ein Pick-by-Watch-System Kommissionierungsleistungen gegenüber herkömmlichen Papierlisten?
}

\section{Ergebnisse einer laborexperimentellen Studie}

\author{
Torsten J. Gerpott ${ }^{1} \cdot$ Ahmet Kurt $^{1}$
}

Online publiziert: 20 . August 2020

(c) Der/die Autor(en) 2020

\section{Zusammenfassung}

Die vorliegende Studie vergleicht ein Pick-by-Watch (PbW)-System mit dem Pick-by-Paper (PbP)-Verfahren im Hinblick auf fünf objektiv und sieben subjektiv gemessene Dimensionen von Kommissionierleistungen in einem Laborexperiment mit 55 Teilnehmern. Uni- und multivariate Analysen sprechen dafür, dass (1) die Kommissioniergeschwindigkeit bei PbW im Vergleich zu PbP signifikant niedriger ausfällt und (2) Fehler bei PbW nicht seltener auftreten als bei PbP. Hingegen schneidet PbW bei subjektiven Messungen von vier Reaktionsaspekten (empfundene Anforderungen, Leistungszufriedenheit, Unterstützung, Nutzerfreundlichkeit) besser ab als PbP. Aus diesem Ergebnismuster werden Schlussfolgerungen für Unternehmenspraxis und die Forschung abgeleitet.

Praktische Relevanz Anstelle von herkömmlichen Kommissionierlisten auf Papier (Pick-by-Paper; PbP) testen Unternehmen in der Lagerbewirtschaftung zunehmend informationsdarstellende digitale Assistenzsysteme, da sie sich hiervon Verbesserungen von Kommissionierleistungen versprechen. Eine Variante solcher Assistenzsysteme, Pick-by-Watch (PbW), verwendet vernetzte Armbanduhren mit berührungsempfindlichen Bildschirmen (Smart Watches). Überraschenderweise mangelt es aber bislang an methodisch soliden empirischen Analysen zum Ausmaß, in dem mit PbW-Systemen tatsächlich bessere Kommissionierleistungen erzielt werden als mit papierbasierten Kommissionierlisten. Derartige Befunde sind jedoch erforderlich, um Praktiker dabei zu unterstützen, wirksamere Auswahlentscheidungen bezüglich vernetzter digitaler Assistenzsysteme in der manuellen Kommissionierung zu treffen.

Schlüsselwörter Digitale Assistenzsysteme $\cdot$ Kommissionierung $\cdot$ Lagerbewirtschaftung $\cdot$ Pick-by-Paper $\cdot$ Pick-by-Watch

Univ.-Prof. Dr. Torsten J. Gerpott

torsten.gerpott@uni-due.de

1 Lehrstuhl Unternehmens- und Technologieplanung, Schwerpunkt Telekommunikationswirtschaft, Mercator School of Management, Universität Duisburg-Essen, Lotharstr. 65, 47057 Duisburg, Deutschland 


\title{
Does a pick-by-watch system improve order picking performance compared to conventional paper lists?
}

Results of a laboratory study

\begin{abstract}
The present study designed a lab experiment to empirically compare the performance of a pick-by-watch (PBW) system and a pick-by-paper (PBP) commissioning procedure in terms of five objectively and seven subjectively measured picking performance dimensions collected from a sample of 55 test persons. Uni- and multivariate statistical analyses suggest that (1) picking speed is significantly lower for PBW in comparison to PBP and (2) PBW does not outperform PBP with regard to picking errors. However, PBW fares significantly better than PBP for four subjectively rated performance criteria (workload, performance satisfaction, support, ease of use). From this pattern of results conclusions are drawn for future research and industrial engineering practice.

Practical Relevance In managing warehouse commissioning tasks quite a number of companies currently consider to replace conventional paper picking lists (pick-by-paper; PBP) by networked commissioning assistance systems drawing on digital devices to display and process information because they expect significantly positive picking performance effects from such systems. One variation of digital picking assistance systems, pick-by-watch (PBW), uses ("smart") networked wrist watches with touch sensitive screens. To date, warehouse managers lack solid empirical evidence on the extent to which PBW systems actually outperform traditional paper picking lists. However, such knowledge is sorely needed to support practitioners in improving selection decisions with regard to networked manual commissioning assistance systems.
\end{abstract}

Keywords Digital assistance systems · Order picking - Pick-by-Paper · Pick-by-Watch · Warehouse management

\section{Hintergrund und Zielsetzung der eigenen Studie}

Einen kostenintensiven Bereich der unternehmensinternen Logistik stellt die Kommissionierung dar. Sie umfasst die auftragsbezogene Zusammenstellung einer Teilmenge materieller Güter aus dem Bestand aller Güter in einem Lager (ten Hompel et al. 2018, S. 55-56, 2019, S. 114). Kommissionierungstätigkeiten können automatisiert (Ware-zurPerson) oder manuell (Person-zu-Ware) ausgeführt werden. Ungeachtet der Leistungsfähigkeit etlicher vollautomatisierter Kommissioniersysteme bevorzugen viele Unternehmen die einerseits personalintensivere, aber andererseits weniger investitionsintensive manuelle Methode, nicht zuletzt deshalb, weil Kommissionierprozesse oft komplex und einzelfallspezifisch ausfallen (vgl. Franzke 2018, S. 3-4). Ansätze wie die bestelltagsgleiche Belieferung oder die kundenspezifische Gestaltung von Leistungen (Losgröße 1) führen auch bei manuellen Kommissioniersystemen zu höheren Anforderungen und machen Prozessoptimierungen notwendig (vgl. Funke 2020, S. 108; Funk et al. 2018, S. 213). Daher setzt man sich in der Praxis und Wissenschaft mit Möglichkeiten der Verbesserung der manuellen Kommissionierung u. a. durch den Einsatz informationsdarstellender digitaler Assistenzsysteme auseinander (vgl. etwa Franzke 2018, S. 66-70; Rammelmeier 2017, S. 16; zu einer Systematisierung solcher Systeme siehe Gerpott und Kurt 2020, S. 18-19). Auch diese Systeme erfordern oft erhebliche Investitionen, so dass vor allem viele kleine und mittlere Unternehmen weiter auf ,klassische“ auftragsbezo- gene Papierlisten (Pick-by-Paper (PbP)) setzen (vgl. Franzke 2018, S. 66-70; Händlerbund 2018, S. 4). Allerdings hat der Kosten-/Preisrückgang bei über Funkverbindungen (Wireless Local Area Network (WLAN), öffentlicher Mobilfunk) vernetzte Armbanduhren mit berührungsempfindlichen Bildschirmen (Smart Watches) in der jüngeren Vergangenheit dazu beigetragen, dass entsprechende wenig(er) investitionsintensive „Pick-by-Watch“ (PbW) Systeme in Unternehmen verschiedenster Größe erprobt werden (Bächler 2017; Maienschein 2017; Kasselmann und Willeke 2016; Reichel 2016).

$\mathrm{PbW}$-Systeme zeichnen sich dadurch aus, dass unter Rückgriff auf eine programmierbare Softwareschnittstelle und eine funkgestützte Telekommunikationsverbindung von einem Kommissionierungsleitstand auf eine vom Kommissionierer am Arm getragene Uhr/Smart Watch optimierte und disponierte Auftragsinformationen dem Kommissionierer mitgeteilt werden. Um die Häufigkeit von Kommissionierfehlern zu verringern, ist bei den meisten Systemen nach Entnahme eines Objekts aus dem Lager vom Kommissionierer auf dem Display der Uhr eine am Lagerort angebrachte Codierung als Auslöser für die Bereitstellung von Informationen zu dem nächsten abzuholenden $\mathrm{Ob}$ jekt einzugeben. Anders als $\mathrm{PbP}$ ermöglicht $\mathrm{PbW}$ somit freihändiges Arbeiten (s. Bächler 2017). Zudem kann bei Beschränkung der Menge der auf der Uhr dargestellten Informationen die mentale Beanspruchung von Kommissionierern gegenüber Systemen, die größere Informationsmengen vermitteln (z.B. Datenbrillen), verringert werden (Maienschein 2017). Allerdings kann der kleine Bildschirm 
von Smart Watches dazu beitragen, dass zusätzliche Zeit für ein korrektes Ablesen von Auftragsinformationen benötigt wird oder vermehrt Lesefehler auftreten.

Angesichts dieser „gemischten“ Vorteil-Nachteil-Bilanz von PbW-Systemen überrascht es, dass es bislang kaum wissenschaftliche Studien gibt, die analysieren, inwiefern sich Kommissionierleistungen bei der Nutzung von $\mathrm{PbW}$-Systemen einerseits und von herkömmlichen Papierlisten oder von anderen digitalen Assistenzsystemen andererseits unterscheiden. Stattdessen wurden Kommissionierleistungsanalysen primär für relativ zu $\mathrm{PbW}$ teure Datenbrillen vorgelegt (vgl. Murauer 2019; Thomas et al. 2018; Niemöller et al. 2017; Rammelmeier 2017; Stoltz et al. 2017; Funk et al. 2015). Leistungsvergleiche für Smart-Watch-Systeme wurden - wenn überhaupt - nur für Tätigkeiten in der Produktion oder Montage veröffentlicht (vgl. Bröring et al. 2019; Funk et al. 2018; Aromaa et al. 2016).

Vor diesem Hintergrund verfolgt die eigene Arbeit das Ziel, ein PbW-System mit Papierlisten im Hinblick auf objektive und subjektive Kriterien zur Abbildung von Kommissionierungsleistungsdimensionen/-facetten in einem Laborexperiment empirisch zu vergleichen. Dazu wird in Abschn. 2.1 das experimentelle Studiendesign vorgestellt. Anschließend werden in Abschn. 2.2 die Untersuchungsteilnehmer profiliert und die Operationalisierung der Leistungskriterien beschrieben. Abschn. 3 präsentiert die Leistungsvergleichsergebnisse. Aus den empirischen Befunden werden in Abschn. 4 Schlussfolgerungen für die weitere Forschung und die Unternehmenspraxis gezogen.

\section{Experimentelles Studiendesign}

\subsection{Lageraufbau und Assistenzsysteme}

Für die Untersuchung wurde ein experimentelles Lager mit vier alphabetisch gekennzeichneten Regalzeilen (A-D/ Länge: 4,00m, Tiefe: 0,25m) und zwei Gängen (Breite: $1,16 \mathrm{~m}$ ) in einem Raum der Universität Duisburg aufgebaut (s. Abb. 1). Eine Regalzeile umfasste fünf Ebenen, wobei die unterste und oberste Ebene jeder Zeile zur Erleichterung der Kommissionierung bzw. Vermeidung körperlicher Überforderung von Versuchsteilnehmern mit kleiner Körpergröße freigelassen wurde (Höhe Ober- bzw. Untergrenze der bestückten Regalebenen: 1,57 m bzw. 0,52 m). Auf jeder Ebene wurden vier Böden montiert, die jeweils zwei Plätze für Lagergüter anboten. Für kleinere Lagergüter wurden die Regalböden mit bis zu zwei Sichtlagerkästen besetzt. Größere Artikel, wie z.B. Kopierpapier oder Ordner, wurden ohne Kasten auf einem Regalboden platziert. Insgesamt wurden so 96 Lagerplätze erzeugt (4 Zeilen $\times 3$ Ebenen $\times 4$ Böden $\times 2$ Orte), die mit verschiedenen

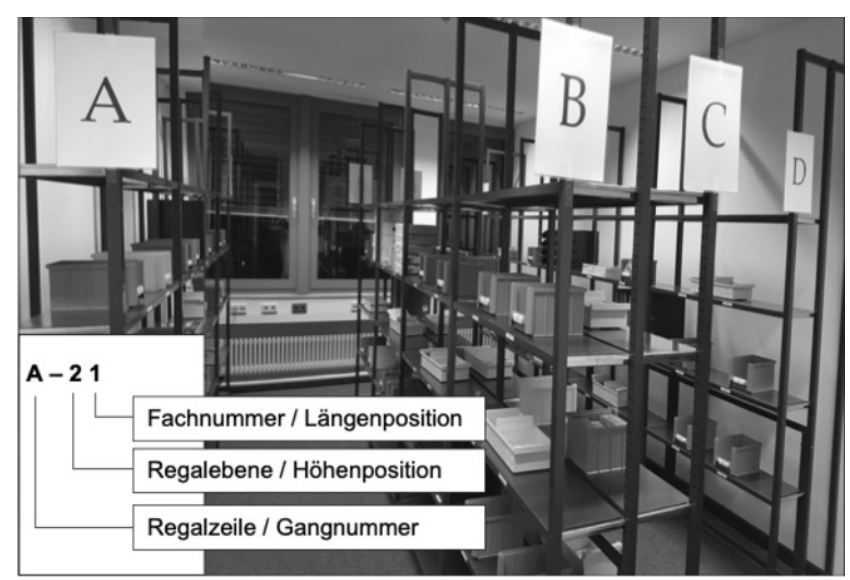

Abb. 1 Aufbau des experimentellen Lagers

Fig. 1 Design of the experimental warehouse

Gütern (z.B. Stifte, Büroklammern) befüllt wurden. Diese Lagerplätze wurden anhand der Merkmale Regalzeile, Regalebene und Fachnummer beschriftet (s. Abb. 1).

Das oben beschriebene Lager wurde von November 2018 bis Februar 2019 zur Datenerhebung betrieben. In dem Lager erhielt jeder Versuchsteilnehmer insgesamt zehn Kommissionieraufträge, von denen sie jeweils fünf mit Papierlisten und mit einem PbW-Assistenzsystem abarbeiteten. Ein Auftrag beinhaltete die Zusammenstellung von verschiedenen Artikeln, von denen jeweils unterschiedliche Mengen aus dem Lager zu entnehmen waren. Für die Aufträge wurden die Zahl der Artikel $(\geq 3$ und $\leq 5)$ sowie die zu entnehmenden Mengen $(\geq 1$ und $\leq 20$ ) zufällig per PC erzeugt. Das zuerst eingesetzte Kommisionierverfahren wurde alternierend variiert, so dass $50 \%$ der Probanden zuerst mit dem PbP( $\mathrm{PbW}-)$ System tätig wurden und danach das $\mathrm{PbW}-(\mathrm{PbP}-)$ Assistenzsystem verwendeten.

Systemübergreifend erhielt jeder Versuchsteilnehmer einen Schiebewagen zum Ablegen der Artikel eines Auftrags. Bestimmte Wegfolgen wurden nicht vorgegeben. Vor Beginn eines Auftrags wurde der Wagen von uns am selben Startort (Anfang des Ganges zwischen den Regalzeilen A und B) positioniert. Ein Auftrag wurde als beendet eingestuft, sobald der Kommissionierer nach Entnahme der Artikel aus den Regalen mit dem Wagen wieder die Startposition erreicht hatte.

Jeder Kommissionierer durchlief folgende sechs Ablaufschritte, die pro Person insgesamt etwa eine Stunde Zeit in Anspruch nahmen:

1. Lektüre Teilnahmeinformationen und Unterzeichnung Einverständniserklärung

2. Erläuterung des Lageraufbaus und des geplanten Versuchsablaufs (inklusive praktische Demonstration von 
Abb. 2 Darstellung der Auftragsinformationen in den Assistenzsystemen

Fig. 2 Presentation of order information in each of the assistance systems

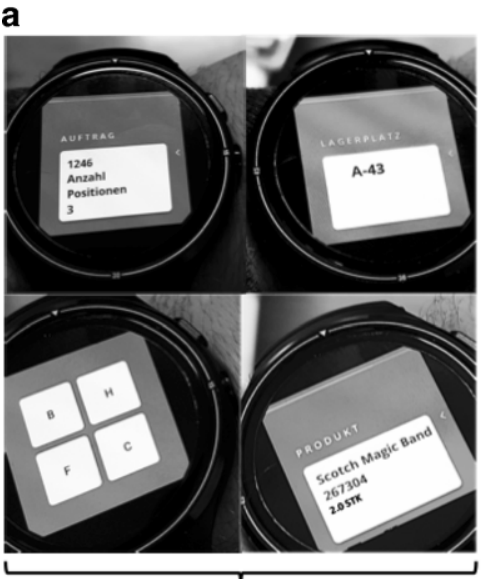

Pick-by-Watch-System b

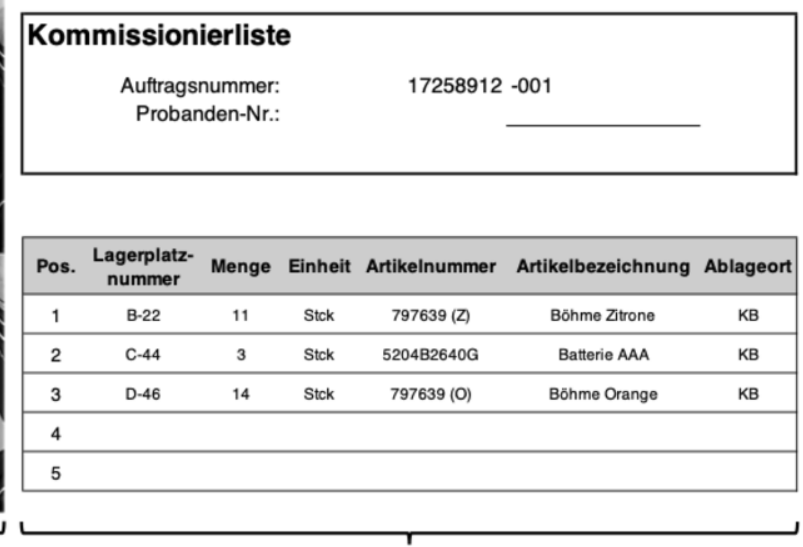

Pick-by-Paper-System drei Aufträgen/Entnahmevorgängen jeweils für $\mathrm{PbW}$ und $\mathrm{PbP}$ )

3. Bearbeitung von fünf Aufträgen mit einem der beiden Kommissionierverfahren

4. Ausfüllen eines Fragebogens zu Merkmalen der Teilnehmer und zur Beurteilung des zuerst genutzten Systems

5. Bearbeitung von fünf Aufträgen mit dem verbleibenden Assistenzsystem

6. Ausfüllen eines Fragebogens zur Beurteilung des anderen Assistenzsystems, das im zweiten Durchgang zum Einsatz kam.

Der Kommissionierungsprozess unter Einsatz der Smart Watch begann mit der Anmeldung des Versuchsteilnehmers an einem Leitstand mittels vorher ausgegebener Zugangsdaten (Benutzername, vierstellige Pin, die der Teilnehmernummer entsprach (z.B. Nr: 07, Pin: 0707)). Anschließend war es dem Probanden möglich, mit einer ersten Wischbewegung auf der Armbanduhrenoberfläche die Zuordnung eines Auftrags (Benennung der Gesamtzahl der zu abzuholenden verschiedenen Artikel) auf die Uhr über das WLAN anzustoßen (s. Abb. 2a).

Mit einer zweiten Wischbewegung wurde für den ersten Artikel dessen Lagerplatz (Regalzeile, Regalebene, Fachnummer) angezeigt. Mit dieser Information konnte sich der Kommissionierer zum Lagerplatz bewegen. Dort war ein dreistelliger alphabetischer Prüfcode (z. B. HBF) angebracht, der vom Kommissionierer über die berührungsempfindliche Oberfläche der Uhr eingegeben wurde. Nach einer korrekten Codeangabe wurden auf der Uhr die Artikelbezeichnung und -nummer sowie zu entnehmende Menge angezeigt. Auf einen falschen Code reagierte die Uhr mit einer Vibration als Hinweis auf einen Fehler und Aufforderung zur erneuten Eingabe des (korrekten) Codes. Die entnommenen Exemplare eines Artikels wurden in einem Behälter auf dem mitgeführten Schiebewagen abgelegt. Danach forderte der Komissionierer mit einer erneuten Wischbewegung auf der Oberfläche der Uhr Daten zum Lagerplatz des nächsten Artikels des Auftrags an. Die Anzeige weiterer Artikel auf der Uhr erfolgte solange bis der letzte Artikel eines Auftrags entnommen war und das Auftragsende in grün auf der Uhr angezeigt wurde. Danach kehrte der Proband mit dem Schiebewagen zum Startort zurück. Anschließend wurde dem Kommissionierer solange ein neuer Auftrag zugewiesen bis fünf Aufträge mit dem PbW-System erledigt waren.

Bei der Pick-by-Paper-Kommissionierung wurden pro Auftrag die Daten in Form einer durchnummerierten Liste auf einem DIN-A4-Blatt zur Verfügung gestellt (s. Abb. 2b). Die zu bearbeitenden fünf Aufträge wurden hintereinander auf einem Klemmbrett befestigt, das dem Probanden übergeben wurde. Dabei wurden die Versuchspersonen darauf hingewiesen, dass (1) ein Ablegen des Klemmbretts untersagt war, um auf eine einhändige Entnahme von Artikeln hinzuwirken, und (2) die Quittierung der Entnahme von Artikeln auf der Liste durch Setzen eines Zeichens (z. B. Haken) gestattet, aber nicht zwingend gefordert war. Das Vorgehen beim Anlaufen der Artikel wurde dem Kommissionierer freigestellt, so dass er sich nicht an die Reihenfolge zu halten hatte, die auf der Papierliste angegeben war. Nach Entnahme sämtlicher Artikel eines Auftrags kehrte der Proband mit dem Schiebewagen zum Startpunkt zurück. Dort wurden die Artikel des Auftrags vom Wagen entfernt. Anschließend wurde der Schiebewagen erneut am Startpunkt positioniert und der nächste Auftrag abgearbeitet. Dieser Prozess wurde wiederholt bis fünf Aufträge bewältigt waren. 


\subsection{Gewinnung und Profilierung der Untersuchungsteilnehmer}

Aufgrund von Beschränkungen hinsichtlich des Forschungsbudgets und der zeitlichen Verfügbarkeit des Raumes, in dem das Lager aufgebaut war, wurde eine kostengünstige und schnelle Rekrutierung von Untersuchungsteilnehmern nach dem Schneeballsystem/-prinzip gewählt (vgl. zu ähnlichen Vorgehensweisen in empirischen Studien zu digitalen Assistenzsystemen in der Arbeitswelt bzw. anderen arbeitswissenschaftlichen Fragen Niemöller et al. 2017, S. 415; bzw. Poller et al. 2016, S. 315-316). In einem ersten Schritt wurden an der Universität, an der die Verfasser tätig sind, Angestellte, Beamte und Studenten auf die Studie durch persönliche Hinweise aufmerksam gemacht und darum gebeten, durch einen Eintrag in eine Online Terminliste eine ernsthafte Bereitschaft zum Durchlaufen des Experimentallagers zu signalisieren. Die so gewonnenen Probanden wurden in einem zweiten Schritt nach ihrer Mitwirkung an dem Kommissionierexperiment aufgefordert, andere Personen auf unsere Untersuchung hinzuweisen und den Link zur Online-Terminvereinbarung für die Kommissionierung an sie weiterzuleiten. Als Anreiz für die Mitwirkung wurde die Verlosung der im Experiment eingesetzten Smart Watch unter allen Teilnehmern versprochen.

Insgesamt konnte auf diese Weise eine (Gelegenheits-) Stichprobe von 55 Personen akquiriert werden. Damit basieren die eigenen Analysen auf einem Stichprobenumfang, der zumeist doppelt so groß ist wie in vergleichbaren empirischen Studien zu Assistenzsystemen in der Kommissionierung (vgl. Stockinger et al. 2020 (31) ${ }^{1}$; Murauer 2019 (23); Thomas et al. 2018 (12); Bächler 2017 (24, 24, 6); Rammelmeier 2017 (17); Funk et al. 2016 (16); Wu et al. 2016 (12); Funk et al. 2015 (16); Wu et al. 2015 (8); Guo et al. 2014 (8); Baumann 2013 (12, 20); Günthner und Rammelmeier $2012(17,6)$; Schwerdtfeger $2010(19,8)$; Günthner et al. 2009 (18, 16); Reif und Walch 2008 (17)). Tab. 1 profiliert unser Sample im Hinblick auf per schriftlicher Befragung gewonnene Angaben (s. Schritt 4 in Abschn. 2.1) zu vier sozio-demographischen Merkmalen (s. Variablen 1-4) sowie vier Charakteristika, die im Zusammenhang mit dem Vergleich von Kommissionierungsleistungen bei Verwendung verschiedener Assistenzsysteme von besonderer Bedeutung sein dürften (s. Variablen 5-8).

Das Alter der Teilnehmer bewegte sich zwischen 18 und 66 Jahren bei einem Mittelwert (Median) von 30,1 $(26,0)$ Jahren. $47,3 \%$ der Versuchspersonen waren Frauen. $50,9 \%$ gaben an, ein (Fach-)Hochschulstudium abge-

\footnotetext{
${ }_{1}^{1}$ Die eingeklammerte Zahl gibt die Zahl der Teilnehmer der jeweiligen Studie an. Wenn mehrere Werte in Klammern aufgeführt werden so bedeutet dies, dass in der Veröffentlichung über mehrere (unabhängige) Erhebungen bzw. Stichproben berichtet wird.
}

Tab. 1 Stichprobenmerkmale $(n=55)$

Table 1 Sample characteristics $(n=55)$

\begin{tabular}{lr}
\hline 1. Alter & \\
- 18-19 Jahre: & \\
- 20-29 Jahre: & $1,8 \%$ \\
- 30-39 Jahre: & $60,0 \%$ \\
- 40-49 Jahre: & $23,6 \%$ \\
- 50-66 Jahre: & $7,3 \%$ \\
2. Geschlecht & $7,3 \%$ \\
- Weiblich: & \\
- Männlich: & $47,3 \%$ \\
3. Hochschulabschluss & $52,7 \%$ \\
- Nein: & \\
- Ja: & $49,1 \%$ \\
4. Nationalität & $50,9 \%$ \\
- Deutsch: & \\
- Nicht deutsch: & $61,8 \%$ \\
5. Kommissioniererfahrung & $38,2 \%$ \\
- Nein: & \\
- Ja: & $87,3 \%$ \\
6. Technologieerfahrung: Smart Devices & \\
- Keine Erfahrung: & $12,7 \%$ \\
- Einige Erfahrung: & \\
- Umfangreiche Erfahrung: & $65,5 \%$ \\
7. Technologieerfahrung: Berührungsgesten & \\
- Keine Erfahrung: & $18,2 \%$ \\
- Einige Erfahrung: & $12,7 \%$ \\
- Umfangreiche Erfahrung: & $69,1 \%$ \\
8. Technologieerfahrung: Smart Watches ${ }^{b}$ & \\
- Keine Erfahrung: & $56,4 \%$ \\
- Einige Erfahrung: & $25,4 \%$ \\
- Umfangreiche Erfahrung: & $18,2 \%$ \\
\hline & \\
\hline & \\
&
\end{tabular}

${ }^{a}$ Mittelwert 30,1 Jahre, Standardabweichung 10,6 Jahre, Median 26 Jahre

${ }^{\mathrm{b}}$ Smart Devices wurden in der Befragung anhand eines Verweises auf „Datenbrillen“ illustriert

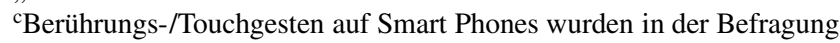
als ,wischen, tippen und zoomen“ präzisiert

${ }^{\mathrm{d}}$ Smart Watches wurden in der Befragung anhand eines Verweises auf Fitness Tracker illustriert

schlossen zu haben. 38,2\% berichteten, nicht die deutsche Staatsangehörigkeit zu besitzen. Im Vergleich zur Grundgesamtheit der volljährigen Bevölkerung in Deutschland sind damit ältere Personen (ab 40 Jahren) ohne Hochschulabschluss und mit deutscher Staatsangehörigkeit in unserer Stichprobe zu selten vertreten (vgl. Statistisches Bundesamt 2019, S. 41-42). Deshalb wurden in einem Teil unserer Leistungsvergleiche u. a. sozio-demographische Charakteristika als Kontrollvariablen einbezogen.

87,3\% der Probanden beantworteten die Frage „Haben Sie bereits früher als Kommissionierer gearbeitet?" mit „Nein“ (s. Variable 5). Der Anteil der Teilnehmer, die erklärten, keine Erfahrung mit Smart Devices bzw. Smart 
Watches zu haben, lag mit 65,5\% bzw. 56,4\% merklich niedriger (s. Variablen 6 und 8). Hingegen berichten $69,1 \%$ der Probanden über umfangreiche Erfahrung mit Berührungsgesten auf Smart Phones zu verfügen. Ein Vergleich der Ausprägungen der im Kontext der Verwendung von $\mathrm{PbW}-\mathrm{Systemen}$ in Kommissionierprozessen speziell relevanten vier Merkmale in unserer Stichprobe mit Werten für die erwachsene Gesamtbevölkerung in Deutschland ist aufgrund des Fehlens einschlägiger veröffentlichter amtlicher Statistiken nicht möglich. Indikativ lässt sich aber festhalten, dass (1) im eigenen Sample Personen mit umfangreicher Erfahrung beim Einsatz berührungsempfindlicher Smart Phone-Oberflächen angemessen erfasst sein sollten ${ }^{2}$ und (2) prima facie kein Grund dafür zu erkennen ist, dass die geringe Kommissioniererfahrung nicht der Ausprägung dieser Variablen in der Grundgesamtheit entsprechenden dürfte.

\subsection{Operationalisierung der Leistungskriterien}

Als abhängige Variablen wurden getrennt für jedes Assistenzsystem zum einen fünf objektive Kriterien, deren Ausprägungen unabhängig von Wahrnehmungen der Versuchspersonen allein über Beobachtungen, die von den das Experiment durchführenden Forschern gemacht/aufgezeichnet wurden, und zum anderen sieben Dimensionen über subjektive Einschätzungen der Teilnehmer gemessen (s. Tab. 2 und 3$).^{3}$

In Anlehnung an einschlägige frühere empirische Forschungsarbeiten zu Assistenzsystemen in der Kommissionierung (z. B. Funk et al. 2016) wurde als erstes objektives Kriterium die Zeit (in Sekunden), die ein Kommissionierer vom Start der Bearbeitung eines Auftrags bis zur Rückkehr mit dem Schiebewagen zum Ausgangspunkt benötigte, gestoppt. Da sich Aufträge hinsichtlich der Zahl der Artikel und der pro Artikel zu entnehmenden Mengeneinheiten un-

\footnotetext{
${ }^{2}$ Repräsentative Befragungen deuten darauf hin, dass Ende 2018 ca. $75-80 \%$ der erwachsenen Wohnbevölkerung in Deutschland ein Smart Phone genutzt hat (Siehe Bitkom 2019, S. 3; Initiative D21 2019, S. 20).

${ }^{3}$ Aus Sicht der klassischen Arbeitswissenschaft lassen sich die fünf objektiv gemessenen Kriterien auch als personenbezogene ,psychophysiologische Beanspruchungsmessung“ oder „Leistungsmaße“ (Schlick et al. 2018, S. 255) im engeren Sinn und die sieben per Fragebogen erhobenen Kriterien als Aspekte der ,subjektiv erlebten Beanspruchung“ (Schlick et al. 2018, S. 265) infolge von Belastungen durch ein Kommissionierungsarbeitssystem charakterisieren. Mit der hier vorgenommenen Unterscheidung zwischen objektiven Leistungs- und (per Befragung erhobenen) Reaktionskriterien wird der eher in der betriebswirtschaftlich-verhaltenswissenschaftlich Forschung (siehe etwa Zerbe 2019, S. 492) üblichen Unterscheidung zwischen tatsächlichem Verhalten (revealed preferences) und Einstellungen (stated preferences) gefolgt. Die objektiven Verhaltens- und subjektiven Reaktionsmaße fassen wir aus Vereinfachungsgründen unter dem Oberbegriff Leistungskriterien zusammen.
}

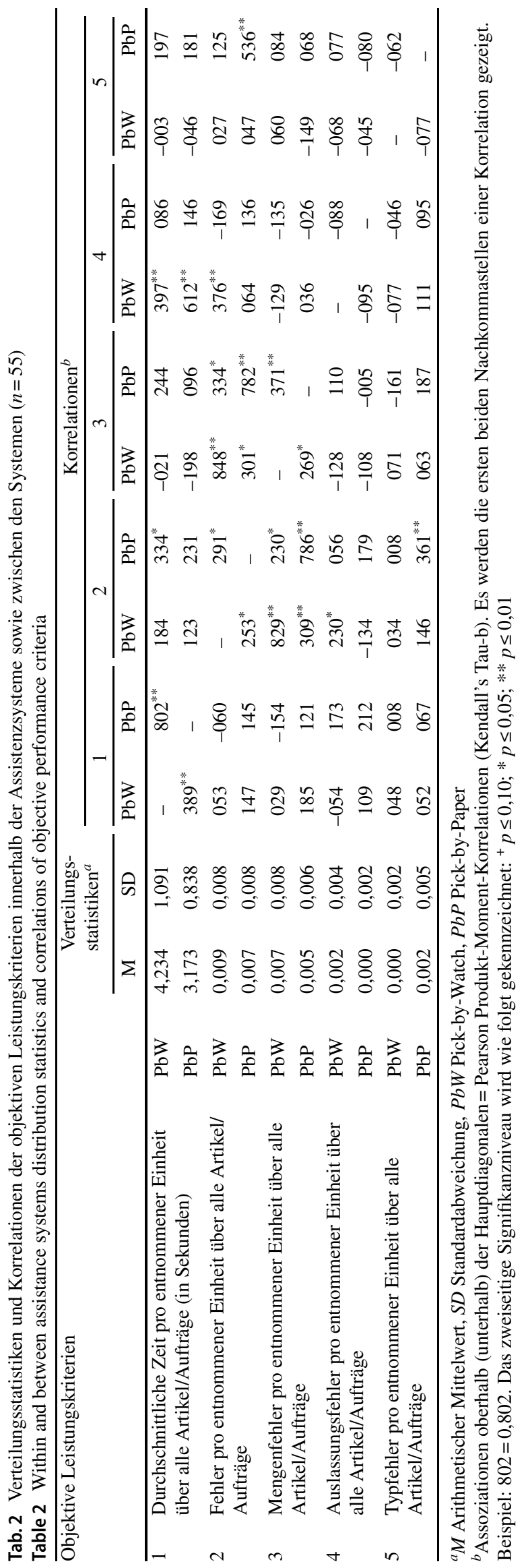




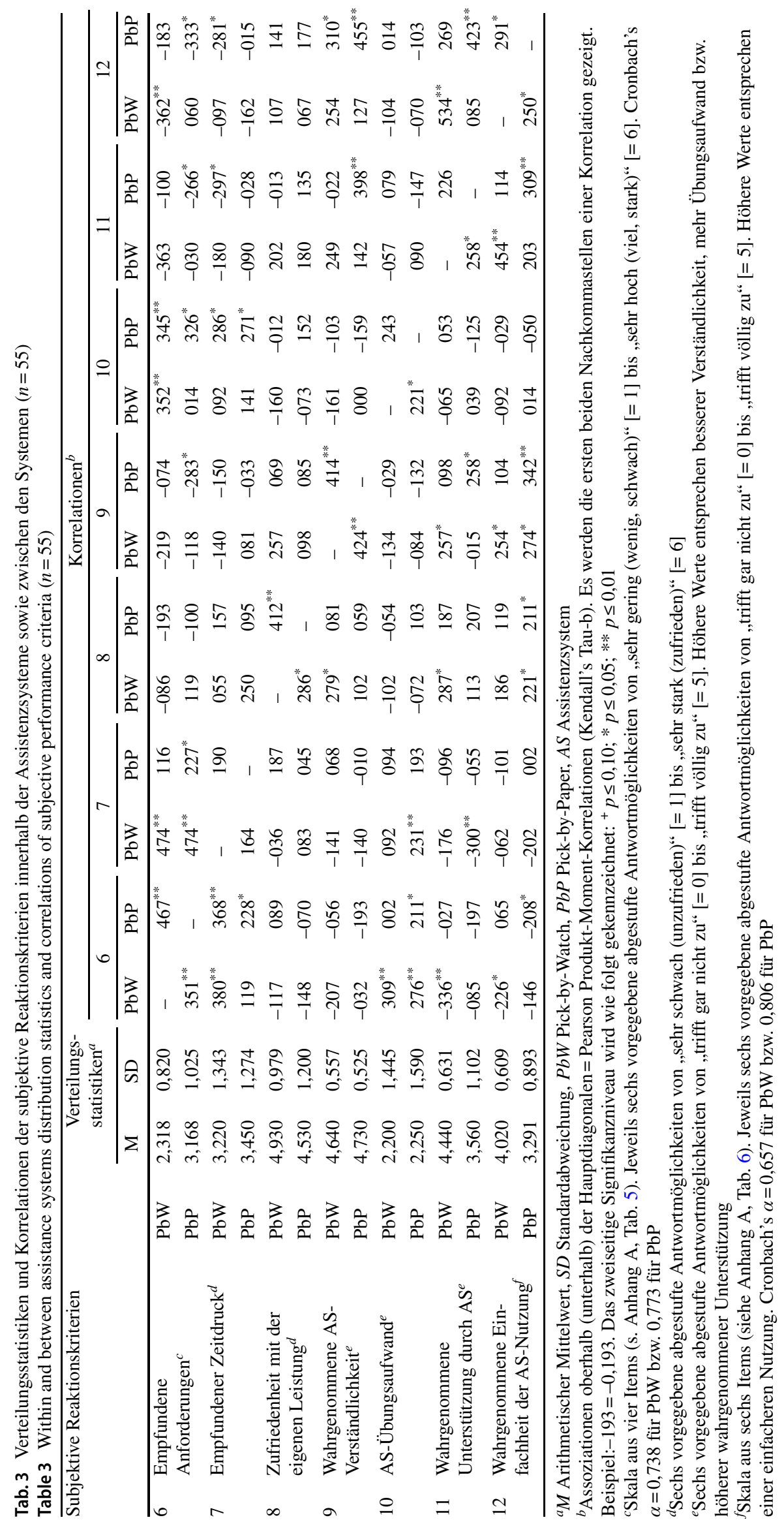


terscheiden, wurde die Zeitvariable pro Auftrag normiert, in dem die gestoppte Zeitdauer durch die Summe der entnommenen Mengeneinheiten aller in einem Auftrag enthaltenen Artikel dividiert wurde. Anschließend wurde der Mittelwert der Zeit pro Mengeneinheit über alle fünf Aufträge berechnet. Gemäß Tab. 2 (s. dort Variable 1) wies die Zeitvariable für $\mathrm{PbW}$ einen Mittelwert von 4,23s und für $\mathrm{PbP}$ einen Durchschnitt von 3,17s auf.

Weiter wurde nach Abschluss der fünf Aufträge pro Assistenzsystem die Zahl jeglicher Kommissionierfehler pro entnommener Mengeneinheit eines Auftrags sowie der Mittelwert der Fehler über die fünf Aufträge bestimmt (s. Variable 2 in Tab. 2). Dabei wurden im Einklang mit relevanten früheren empirischen Studien (z. B. Günthner und Rammelmeier 2012) die drei Fehlertypen Mengenfehler (= Entnahme eines Artikels in einer Menge, die nicht dem Auftrag entspricht), Auslassungsfehler (keine Entnahme eines Artikels, obwohl er Teil des Auftrags war) und Typfehler (Entnahme eines Artikels, der in einem Auftrag nicht enthalten war) unterschieden (s. Variablen $3-5$ in Tab. 2 sowie Anhang B, Tab. 7).

Tab. 2 informiert über deskriptive Verteilungsstatistiken für die fünf objektiven Verhaltenskriterien und deren Korrelationen innerhalb der beiden Assistenzsysteme sowie zwischen den Systemen. Danach benötigten Versuchspersonen, die eine überdurchschnittliche Bearbeitungszeit mit $\mathrm{PbW}$ aufweisen mit dem PbP-System ebenfalls signifikant länger für die Kommissionierung $(r=0,80)$. Dieser Zusammenhang spricht dafür, dass die Bearbeitungszeit auch stark durch Merkmale des Kommissionierers beeinflusst wird. Sowohl bei dem PbW-System als auch bei PbP wird die Fehleranzahl insgesamt am stärksten durch Mengenfehler geprägt ( $r=0,85$ für $\mathrm{PbW} ; r=0,79$ für $\mathrm{PbP}$ ). Die höchsten parametrischen Korrelationen zwischen dem Zeitkriterium und den vier Fehlervariablen waren bei $\mathrm{PbW}$ für Auslassungsfehler $\mathrm{zu}$ beobachten $(r=0,40)$ und bei $\mathrm{PbP}$ für die gesamte Fehlerzahl $(r=0,23)$. Da jedoch die entsprechenden nicht-parametrischen Rangkorrelationen nicht signifikant ausfielen ( $r=-0,05$ für $\mathrm{PbW} ; r=0,15$ für $\mathrm{PbP}$ ), ist $\mathrm{zu}$ vermuten, dass die parametrischen Korrelationen durch Verteilungsanomalien (z.B. Ausreißer) der Variablen stark verzerrt wurden und deshalb mit der Zeitdauer sowie Fehler(typen)zahl eher voneinander unabhängige Dimensionen erfasst wurden.

Die subjektiven Reaktionskriterien wurden getrennt für jedes Assistenzsystem über Einschätzungen der Versuchsteilnehmer gemessen, die sie direkt nach Bearbeitungsende der fünf Aufträge in einem Fragebogen für das unmittelbar zuvor genutzte System abgaben. Hierbei kamen insgesamt 15 Items zum Einsatz, die unter Rückgriff auf die Ergebnisse von Faktor- und Reliabilitätsanalysen zu sieben Dimensionen verdichtet wurden (s. Tab. 3).
Das erste Kriterium empfundene Anforderungen umfasst den Durchschnitt der Antworten zu vier Items zur (aus objektiven Belastungen resultierenden) erlebten geistigen und körperlichen Beanspruchung durch das verwendete Assistenzsystem (vgl. zur konzeptionellen Einordnung dieser Beanspruchungsfelder Schlick et al. 2018, S. 266). Die Items wurden der Erhebung von Bächler (2017) entnommen (s. Variable 6 in Tab. 3 sowie Anhang A, Tab. 5). Die interne Konsistenzreliabilität dieser Skala erreichte mit Cronbach $\alpha$-Werten von 0,74 für $\mathrm{PbW}$ und 0,77 für $\mathrm{PbP}$ ein zufriedenstellendes Niveau. Die zweite bzw. dritte subjektive Reaktionsdimension ist die Stärke des wahrgenommenen Zeitdrucks bei der Auftragsbearbeitung mit einem System bzw. das Ausmaß der Zufriedenheit mit dem Grad der Aufgabenerfüllung/eigenen Leistung nach Einsatz des jeweiligen Systems (s. Variablen 7 und 8 in Tab. 3). Die beiden Items stammen ebenfalls aus der Studie von Bächler (2017). Die übrigen über insgesamt neun Items operationalisierten subjektiven Leistungsfacetten 9-12 in Tab. 3 wurden in Anlehnung Rammelmeier (2017) formuliert. Das Kriterium Verständlichkeit (s. Variable 9 in Tab. 3) wurde mittels des Zustimmungsgrades zu der Aussage „Das Assistenzsystem ist in kurzer Zeit verstehbar" gemessen. Die Variable Übungsaufwand (s. Variable 10 in Tab. 3) gibt die Reaktion der Probanden auf das Item „Das Arbeiten mit dem Assistenzsystem erfordert Übung“ wieder. Der Leistungsaspekt Unterstützung (s. Variable 11 in Tab. 3) wurde über das Item „Das Assistenzsystem hat mich bei der Bearbeitung der Kommissionieraufträge unterstützt" operationalisiert. Die Einfachheit der Assistenzsystemnutzung (s. Variable 12 in Tab. 3) wurde über den Mittelwert von sechs Items erfasst, die Anhang A, Tab. 6 dokumentiert. Das Cronbach $\alpha$ belief sich für diese Skala auf 0,66 für das PbW-System und 0,81 für das PbP-System. Die interne Konsistenzreliabilität der Skala ist somit für jedes der beiden Assistenzsysteme akzeptabel.

Die höchste paarweise parametrische Korrelation innerhalb der sieben subjektiven Reaktionsdimensionen betrug für das $\mathrm{PbW}$-System 0,53 (Unterstützung und Nutzungseinfachheit, $r_{11-12}$ in Tab. 3) und für das PbP-System 0,46 (Verständlichkeit und Nutzungseinfachheit, $r_{9-12}$ in Tab. 3). Demnach überlappen sich einige der sieben subjektiven Reaktionsaspekte für $\mathrm{PbW}$ bzw. PbP zwar mit einem gemeinsamen Varianzanteil von bis zu $28 \%$ bzw. $21 \%$ erkennbar. Das Ausmaß dieser Überlappung ist aber noch so niedrig, dass eine getrennte Betrachtung der Aspekte als im Kern keineswegs kongruente Reaktions- bzw. Leistungsdimensionen sinnvoll ist.

Von den 35 paarweisen parametrischen Korrelationen zwischen den fünf objektiven und sieben subjektiven Kriterien waren für das $\mathrm{PbW}-$ System $97 \%$ und für das PbPSystem 91\% nicht auf dem 5\%-Niveau signifikant. Eine signifikante (negative) Assoziation wurde allerdings für das 
$\mathrm{PbW}$-System zwischen dem empfundenen Zeitdruck und der Bearbeitungszeit pro Mengeneinheit (s. $r_{1-7}=-0,31$ in Anhang B, Tab. 7) beobachtet. Beim PbP-System korrelierte der empfundene Zeitdruck hingegen positiv mit der Bearbeitungszeit und der Zahl der Typfehler (s. $r_{1-7}=0,31$ und $r_{5-7}=0,35$ in Anhang B, Tab. 7). Diese Befunde deuten darauf hin, dass ein bei PbW als stark empfundener Zeitdruck zu einer schnelleren Bearbeitung von Kommissionieraufträgen bei $\mathrm{PbW}$ (und $\mathrm{PbP}$ ) beiträgt, ohne die $\mathrm{PbW}$ Leistungsqualität zu beeinträchtigen, während bei $\mathrm{PbP}$ sich wahrgenommener „Zeitstress“ eher leistungsabträglich auswirkt. Alles in allem ist aber festzustellen, dass in unserer Stichprobe mit den fünf objektiven Indikatoren einerseits und den sieben subjektiven Kriterien andererseits unterschiedliche Leistungsfacetten von Assistenzsystemen in der Kommissionierung abgebildet werden.

\section{Empirische Vergleichsergebnisse}

Die statistische Signifikanz der Mittelwertunterschiede der fünf objektiven und subjektiven Kriterien als Funktion des bei der Kommissionierung verwendeten Assistenzsystems wurde mittels parametrischer $t$-Tests für abhängige Stichproben bzw. Messwiederholungen geprüft. Zusätzlich wurden Wilcoxon-Vorzeichen-Rangtests für verbundene Stichproben durchgeführt, um zu erkennen, inwiefern sich die Mediane der 12 Kriterien bei Einsatz eines PbW-Systems von denen bei einer PbP-Kommissionierung unterscheiden. Tab. 4 dokumentiert Ergebnisse der insgesamt 24 Tests.

Von den fünf objektiven Leistungsdimensionen sind für die drei Kriterien Bearbeitungszeit, Mengen- und Auslassungsfehler auf dem 10\%-Niveau (2-seitig) signifikante Unterschiede gleichermaßen bei Mittelwert- und Medianvergleichen $\mathrm{zu}$ beobachten (s. Variablen 1,3 und 4 in Tab. 4). Die drei Leistungsaspekte waren für das $\mathrm{PbW}$ System durchweg schlechter ausgeprägt als für das $\mathrm{PbP}-$ Verfahren. Hingegen unterscheiden sich die beiden Assistenzsysteme bezüglich der gesamten Fehlerzahl (aggregiert über die drei Fehlertypen) und der Zahl der Typfehler nicht signifikant.

Mit Blick auf die sieben subjektiven Reaktionsaspekte ist Tab. 4 zu entnehmen, dass für vier Kriterien signifikante Unterschiede auftreten: Das PbW-System wurde bezüglich der Anforderungen, Leistungszufriedenheit, Unterstützung des Kommissionierprozesses und Einfachheit der Nutzung (s. Variablen 6, 8, 11 und 12 in Tab. 4) jeweils besser beurteilt als das PbP-Verfahren. Hingegen gab es keine Einstufungsunterschiede zwischen $\mathrm{PbW}$ und $\mathrm{PbP}$ im Hinblick auf die Kriterien Zeitdruck, Verständlichkeit und Übungsaufwand (s. Variablen 7, 9 und 10 in Tab. 4).

Um potentielle Einflüsse (1) sozio-demographischer Probandenmerkmale (2) ihrer Erfahrung mit Kommissionier- aufgaben sowie Geräten, die ähnliche Nutzerschnittstellen wie das PbW-System haben, und (3) der Einsatzfolge der beiden Assistenzsysteme im Laborexperiment auf die bivariaten Mittelwertvergleichsergebnisse zu erkunden, wurden die acht Tab. 1 zu entnehmenden Probandenmerkmale sowie die Sequenz der Assistenzsysteme im Laborexperiment in (Ko-)Varianzanalysen für Messwiederholungen bei jedem der 12 Leistungskriterien als Kontrollvariablen/ Kovariaten eingeführt. In diesen Kovarianzanalysen wurde neben den Haupteffekten der Kontrollvariablen auch ermittelt, inwiefern sich Zusammenhänge zwischen Kontrollvariablen und einer Leistungsdimension in Abhängigkeit vom verwendeten Assistenzsystem unterscheiden (Interaktionsterm Kontrollvariable $\times$ Assistenzsystem). Im Sinn eines induktiven ,,model trimming“ wurden dann für jedes Leistungskriterium sämtliche Interaktionsterme eliminiert, die nicht mindestens auf dem 5\%-Niveau statistisch signifikant waren und anschließend nur noch die Signifikanz der Haupteffekte der Kontrollvariablen untersucht.

Wie den mit „signifikante Kontrollvariablen $(p \leq 0,05)$ “ überschriebenen Spalten in Tab. 4 zu entnehmen ist, erreichten von den 108 Interaktionstermen (9 Kontrollvariablen $\times 12$ Leistungskriterien) fünf sowie von den 108 Haupteffekten nur ein Effekt statistische Signifikanz. Um die Interpretation der fünf signifikanten Moderatorfälle zu erleichtern, werden sie in Abb. 3 visualisiert und im Folgenden beschrieben.

Der erste als statistisch signifikant identifizierte Interaktionseffekt zwischen dem Assistenzsystemtyp und den Kontrollvariablen betrifft die Erfahrung mit Smart Watches $(p \leq 0,009$; s. Tab. 4): Bei PbW führt die frühere Nutzung von vernetzten berührungsempfindlichen Armbanduhren zu einer deutlich stärkeren Reduktion der Kommissionierzeit als bei PbP (s. Abb. 3a). Demnach lassen sich Erfahrungen, die allgemein mit Smart Watches in anderen Anwendungssituationen gewonnen wurden, auf $\mathrm{PbW}$-unterstützte Kommissioniertätigkeiten effizienzsteigernd übertragen. Hingegen war in unserer Stichprobe für die Kontrollvariable Kommissioniererfahrung (s. Variable 5 in Tab. 1) bei keinem Leistungskriterium ein signifikanter Interaktionsoder Haupteffekt festzustellen. Dieser Befund kann zum einen daraus resultieren, dass unsere Stichprobe nur sieben Probanden mit Kommissioniererfahrung beinhaltete. Zum anderen deutet er darauf hin, dass nicht Kommissioniererfahrung im Allgemeinen, sondern eher die spezielle Vertrautheit mit dem als Assistenzsystem verwendeten Endgerätetyp die Kommissioniergeschwindigkeit erhöht.

Die zweite statistisch signifikante Wechselwirkung tritt zwischen den Assistenzsystemtyp und dem formalen Bildungsniveau von Probanden auf ( $p \leq 0,027$; s. Tab. 4): Teilnehmer mit Hochschulabschluss begehen mit Smart-WatchUnterstützung weniger Mengenfehler als Kommissionierer ohne Hochschulabschluss. Hingegen ist bei dem PbP-Ver- 


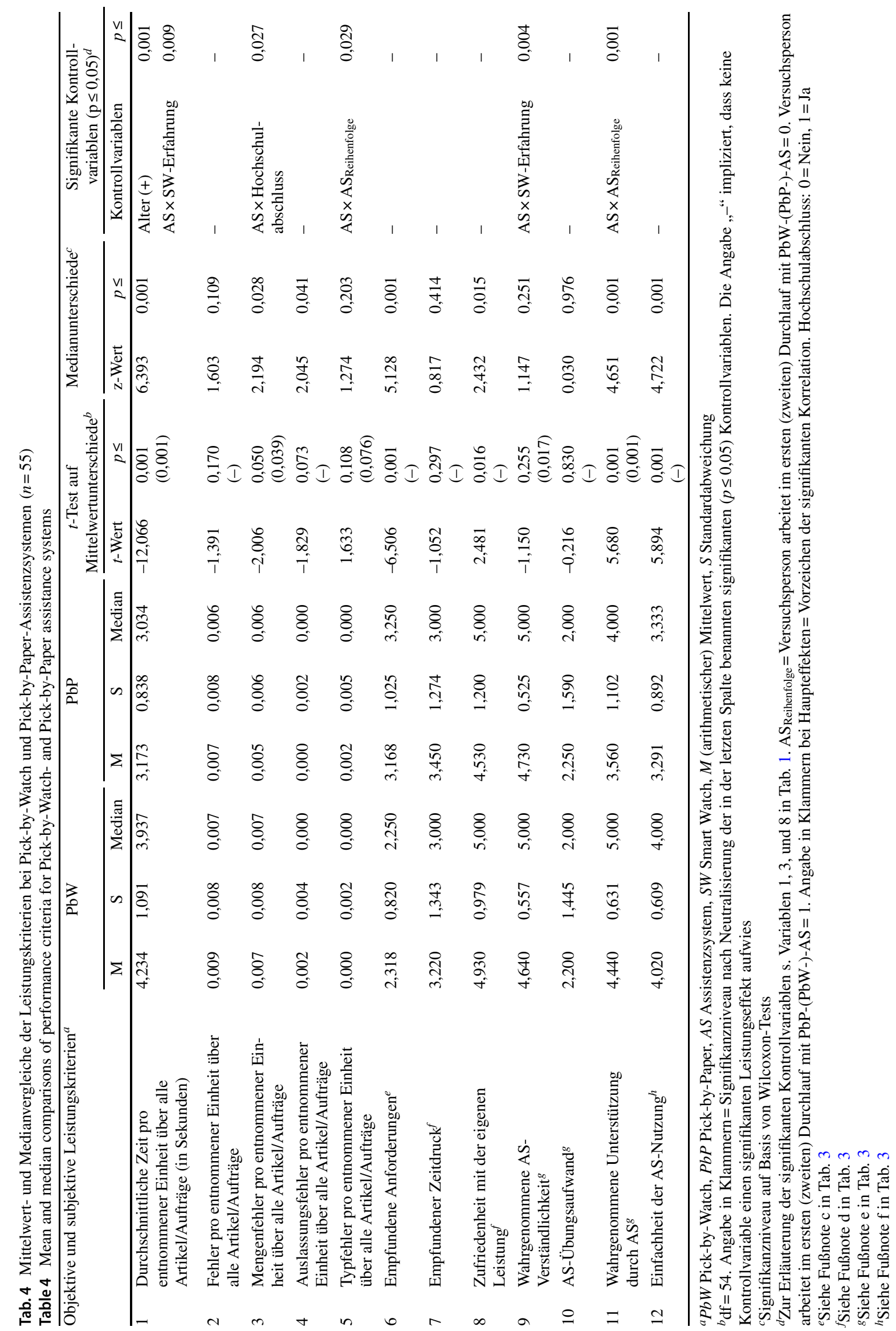


a

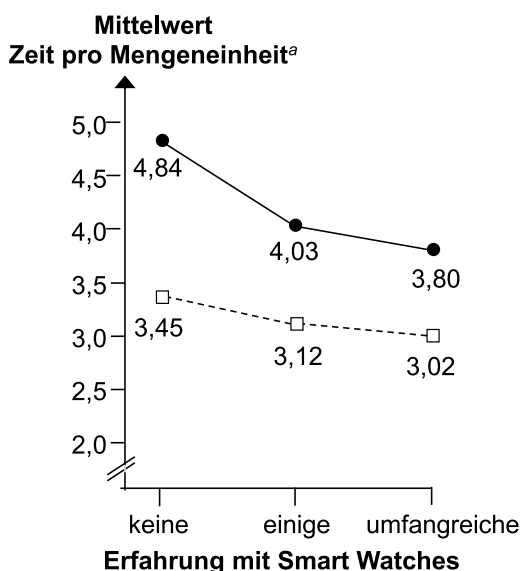

d

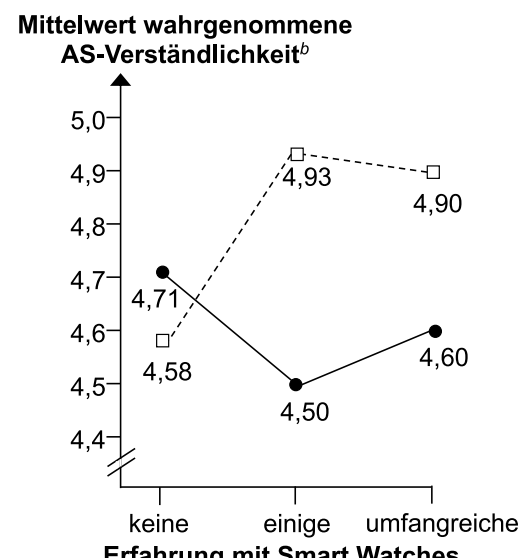

b

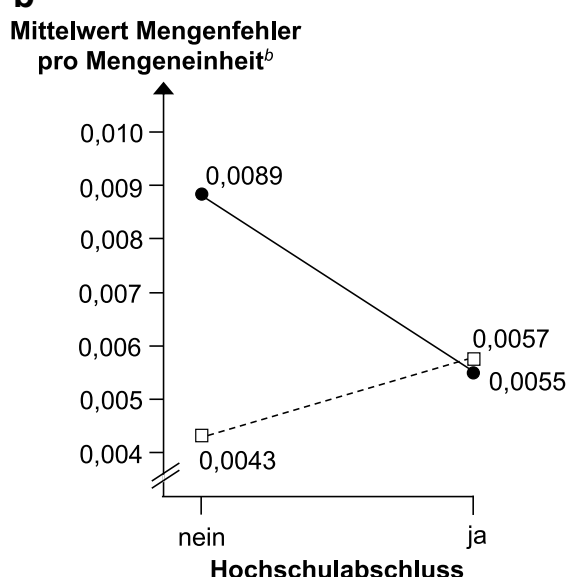

e

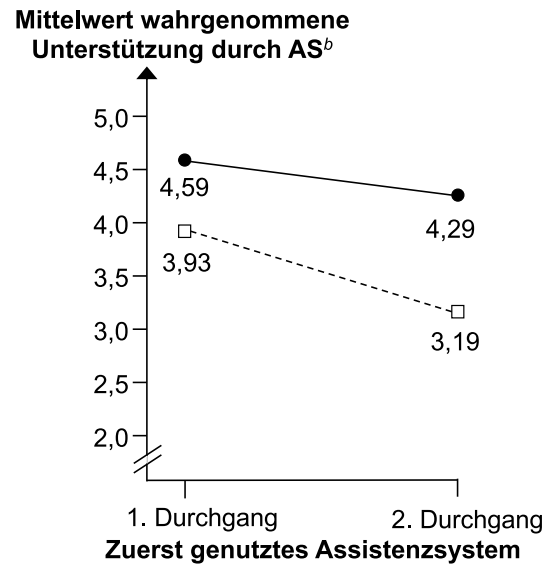

c

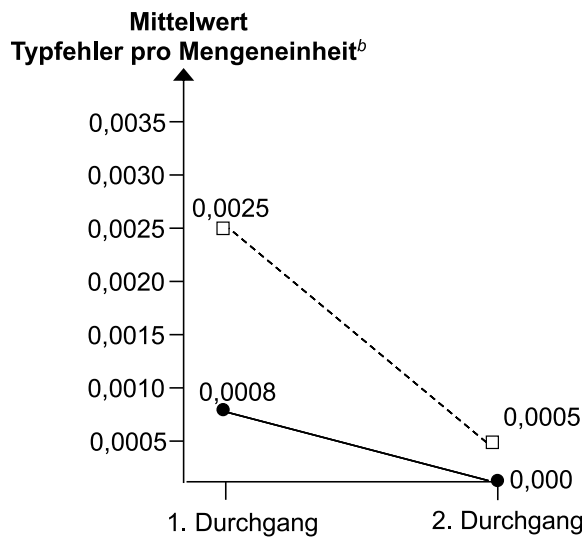

Zuerst genutztes Assistenzsystem

Abb. 3 Visualisierung von mindestens auf dem 5\%-Niveau signifikanten Interaktionen zwischen Kontrollvariablen und Assistenzsystemtyp $(n=55)$. Die obere linke Grafik (a) zeigt die geschätzten Subgruppen-Mittelwerte des objektiven Leistungskriteriums 1 gemäß Tab. 2 für den Fall, dass der Wert der Kontrollvariable Alter gemäß Tab. 1 dessen arithmetischen Durchschnitt entspricht. Die Grafiken b-e zeigen die beobachteten Subgruppen-Mittelwerte für die objektiven Leistungskriterien 3 und 5 gemäß Tab. 2 sowie die subjektiven Leistungskriterien 9 und 11 gemäß Tab. 3

Fig. 3 Visualization of significant $(p \leq 0.05)$ interactions between control variables and assistance system type $(n=55)$

fahren die Mengenfehlerzahl weitgehend unabhängig vom formalen Bildungsniveau (s. Abb. 3b). Sieht man einen Hochschulabschluss als Indikator dafür an, ob eine Person über Fähigkeiten zur Bewältigung kognitiv komplexer Aufgaben verfügt, dann spricht der zweite Effekt dafür, dass Kommissionierer mit geringem formalen Bildungsniveau durch ein PbW-System eher kognitiv überfordert werden als bei Verwendung herkömmlicher Papierlisten.

Der dritte signifikante Interaktionseffekt ist zwischen dem Assistenzsystemtyp und der Reihenfolge der Nutzung der beiden Assistenzsysteme im Laborexperiment zu beobachten ( $p \leq 0,029$; s. Tab. 4): Bei Probanden, die zuerst mit dem PbP-Verfahren arbeiten, treten Typfehler in größerem Ausmaß auf als bei Teilnehmern, die im zweiten Durchgang auf Papierlisten zurückgriffen, nachdem sie zuvor im ersten Durchgang das PbW-System genutzt hatten.
Demgegenüber ist die Typfehlerzahl bei $\mathrm{PbW}$ unabhängig davon, ob das System einem Teilnehmer im ersten oder zweiten Durchlauf zur Verfügung stand (s. Abb. 3c). Dieses Ergebnis läßt sich dadurch erklären, dass das $\mathrm{PbW}$ System durch die Notwendigkeit der Eingabe von Codes bei einer Artikelentnahme während der Abarbeitung eines Kommissionierauftrags so gestaltet war, dass Typfehler unabhängig davon, ob unmittelbar zuvor bereits Erfahrungen mit PbP gewonnen wurden oder nicht, nahezu vollständig ausgeschlossen waren.

Die vierte signifikante Wechselwirkung besteht zwischen dem Assistenzsystemtyp und der Kontrollvariablen Erfahrung mit Smart Watches ( $p \leq 0,004$; s. Tab. 4): Die Verständlichkeit des PbP-Verfahrens wird dann wesentlich besser beurteilt, wenn ein Kommissionierer bereits Erfahrungen mit Smart Watches aufweist. Die Einschätzung der Ver- 
ständlichkeit des $\mathrm{PbW}$-Systems ist hingegen weitgehend unabhängig davon, ob ein Teilnehmer schon über Erfahrungen mit vernetzten Armbanduhren verfügt oder nicht (s. Abb. 3d). Eine Post-hoc-Erklärung für dieses Resultat ist, dass Probanden mit Smart-Watch-Erfahrung aufgrund früherer negativer Erlebnisse mit der Verständlichkeit solcher Geräte das PbP-Verfahren vor dem Hintergrund dieses Maßstabs milder einstufen könnten als Teilnehmer ohne Smart-Watch-Erfahrung.

Der fünfte signifikante Interaktionseffekt betrifft neben dem Assistenzsystemtyp wieder die Sequenz der Nutzung der beiden Assistenzsysteme im Experiment $(p \leq 0,001$; s. Tab. 4): Die wahrgenommene Kommissionierunterstützung durch $\mathrm{PbW}$ wird nicht davon beeinflusst, ob ein Teilnehmer unmittelbar zuvor bereits Erfahrungen mit PbP gewonnen hat oder nicht. Hingegen fällt die Beurteilung des Ausmaßes der Unterstützung durch PbP dann deutlich schlechter aus, wenn die Probanden zuvor bereits das PbWSystem kennengelernt haben (s. Abb. 3e). Hier kann vermutet werden, dass die unmittelbar vor dem PbP-Einsatz erlebte starke Unterstützung durch das PbW-System den internen Referenzpunkt der Probanden bei Unterstützungsbeurteilungen verschiebt und in der Folge die Beurteilung der PbP-Unterstützung strenger bzw. schlechter ausfällt.

Der einzige signifikante Haupteffekt einer Kontrollvariablen ist in unserer Stichprobe für das Lebensalter festzustellen ( $p \leq 0,001$; s. Tab. 4): Mit dem Alter steigt die pro Artikel/Auftrag benötigte Zeit. Dieser Zusammenhang ist plausibel, da das Alter zumindest mit der körperlichen Leistungsfähigkeit/Schnelligkeit der Probanden und möglicherweise auch mit deren Leistungsmotivation/,Ehrgeiz“ negativ korreliert sein dürfte.

Insgesamt sprechen die Ergebnisse aber dafür, dass die zwölf Kommissionierleistungskriterien durch soziodemographische Merkmale, Erfahrungen mit Kommissionieraufgaben sowie mit Smart Watches oder ähnlichen Geräten kaum beeinflusst werden und die in bivariaten Analysen herausgearbeiteten Leistungsunterschiede/-ähnlichkeiten der beiden Kommissionierverfahren auch nach Berücksichtigung der Kontrollvariablen Bestand haben.

\section{Schlussfolgerungen}

Die vorgelegte Studie erweitert die arbeits- und betriebswirtschaftliche Literatur zu Assistenzsystemen bei manuellen Kommissioniertätigkeiten dadurch, dass sie erstmals für ein $\mathrm{PbW}$-System systematisch in einem Laborexperiment mit 55 Teilnehmern die Ausprägungen verschiedener mittels objektiver Verfahren und über Selbsteinstufungen subjektiv gemessener Aspekte von Kommissionierleistungen erfasst und sie zudem mit den Leistungswerten vergleicht, welche die gleichen Probanden mit dem herkömmlichen
PbP-Verfahren erreichen. Alles in allem werden in unserer Stichprobe mit dem PbW-System nicht weniger Fehler begangen als bei der Kommissionierung mit Papierlisten. Hinsichtlich der Kommissioniergeschwindigkeit werden mit PbP sogar signifikant bessere Ergebnisse erzielt als mit PbW. Für die Praxis bedeuten diese Befunde, dass man nicht davon ausgehen darf, mit dem Rückgriff auf ein ,,modernes“ digitales Assistenzsystem in der manuellen Kommissionierung gewissermaßen automatisch erhebliche Leistungsverbesserungen zu bewirken. Notwendig ist vielmehr ein sorgfältiges Management der ,,kritischen Erfolgsfaktoren“ solcher Systeme, zu denen neben technischen Aspekten auch die Organisation des Einführungsprozesses unter systematischer Einbindung der Aufgabenträger/Anwender gehört (s. Winkler und Zinsmeister 2019, S. 545).

Für das unerwartete, relativ schwache Abschneiden von $\mathrm{PbW}$ gegenüber $\mathrm{PbP}$ in unserem Laborexperiment gibt es drei, sich ergänzende Erklärungen. Erstens wurde den Teilnehmern das PbW-System vor dessen Nutzung zwar detailliert erläutert, aber ihnen nicht die Möglichkeit eingeräumt, es vor dem quasi echten Einsatz länger praktisch auszuprobieren (vgl. zur generellen Tendenz von Unternehmen in Deutschland beim Einsatz von am Körper tragbaren digitalen Assistenzsystemen kaum Anlernmaßnahmen zu ergreifen Pfeiffer et al. 2016, S. 105-106). In zukünftigen Untersuchungen sollte deshalb erkundet werden, ob die angestrebte Geschwindigkeitserhöhung und Fehlerverminderung durch $\mathrm{PbW}$ gegenüber $\mathrm{PbP}$ sich dann einstellt, wenn Aufgabenträger mit mehr Aufwand trainiert werden bevor sie im Alltag mit einem PbW-System kommissionieren. Zweitens war die Kommissionieraufgabe in unserem Experiment nicht sehr komplex, da das Lager nur zwei Gänge und die Aufträge nicht mehr als fünf Artikel umfassten. Deshalb ist nicht auszuschließen, dass mit PbWSystemen im Vergleich zu Papierlisten merklich schneller und weniger fehlerbehaftet kommissioniert wird, wenn diese Systeme in räumlich großen Lagern und bei Aufträgen mit einer höheren Zahl von Entnahmepositionen eingesetzt werden. Auch diese Möglichkeit sollte in der weiteren Forschung beleuchtet werden, indem Kommissioniergeschwindigkeit und -fehler von $\mathrm{PbW}$-Systemen und anderen digitalen Assistenzsystemen (z.B. vernetze Datenbrillen oder Kopfhörer) sowie $\mathrm{PbP}$ an realen Standorten großer Lagerbetreiber vergleichend analysiert werden. Drittens sind der Größe des Bildschirms der eingesetzten Armbanduhr durch den Gerätetyp bedingt enge Grenzen gesetzt, die zu Ablese- und Eingabeschwierigkeiten beigetragen haben könnten (vgl. Dehghani und Kim 2019; Hara et al. 2015). Deshalb sollte weiter untersucht werden, inwiefern die Größe der Ablese-/Eingabefläche von Smart-Watch-Systemen sich signifikant auf Kommissioniergeschwindigkeit und -fehler auswirken. 
Bei den sieben durch Selbsteinstufungen erfassten Reaktionsdimensionen werden erlebte Anforderungen, Leistungszufriedenheit, Unterstützung und Nutzerfreundlichkeit des PbW-Systems signifikant besser beurteilt als das PbPVerfahren. Zugleich schneidet das PbW-System gegenüber Papierlisten hinsichtlich der Kriterien Zeitdruck, Verständlichkeit und Übungsaufwand in unserem Laborexperiment nicht signifikant schlechter ab. Gegen die Verallgemeinerbarkeit dieser relativ positiven Wahrnehmungen von $\mathrm{PbW}$ lässt sich anführen, dass die „Begeisterung“ für vernetze Armbanduhren negativ mit dem Lebensalter korreliert ist (vgl. für viele Beh et al. 2019; Park 2020), so dass die besseren Werte von PbW bei vier subjektiven Leistungsaspekten durch das niedrige Alter der Probanden in unserem Versuch (61,8\% jünger als 30 Jahre; s. Tab. 1) hervorgerufen worden sein könnten. In der Praxis werden in der Kommissionierung zwar häufig Studenten eingesetzt. Dennoch ist zu empfehlen, die Haltbarkeit unserer Resultate im Hinblick auf die sieben subjektiv erfassten Leistungsaspekte von Kommissionierverfahren in Stichproben zu prüfen, die in sozio-demographischer Hinsicht und bezüglich der vorhandenen Kommissioniererfahrung repräsentativ(er) für Aufgabenträger in bestimmten Branchen (z. B. Versandhandel) sind und die zwecks Erhöhung der Teststärke einen größeren Umfang als das eigene Sample haben.

Die in der vorgelegten Studie analysierten zwölf abhängigen Kriterien decken zwar behavioristisch-leistungsbezogene sowie empfindungsbezogene, nicht aber physiologisch-organismische Beanspruchungsaspekte ab (vgl. zur Unterscheidung der drei Aspekte Schlick et al. 2018, S. 26-28). In der weiteren Forschung sollten deshalb zusätzlich physiologische Wirkungen des Einsatzes von $\mathrm{PbW}$, anderen digitalen Systemen zur Unterstützung von Kommissionierungarbeitsplätzen und Papierlisten vergleichend erkundet werden. Angesichts dessen, dass bei solchen digitalen Systemen informatorische Belastungen und Beanspruchungen herausragende Bedeutung haben, liegt es hier nahe, Blickaufzeichnungen (Eye-Tracking; vgl. Blake 2013, S. 371-382; Schlick et al. 2018, S. 260) in den Vordergrund zu rücken, um den Informationsgehalt von Anzeigen digitaler Assistenzsysteme in der Kommissionierung $\mathrm{zu}$ optimieren.

In Wissenschaft und Praxis tritt im Zusammenhang mit dem Einsatz von vernetzten digitalen Assistenzsystemen in verschiedenen betrieblichen Funktionseinheiten (z. B. Produktion, Wartung, Kundendienst) nicht selten eine „naive“ Technikeuphorie auf. Nach unseren Erkenntnissen ist sie zumindest für Smart Watches in der Kommissionierung nicht uneingeschränkt gerechtfertigt. Damit steht die arbeitswissenschaftliche Forschung vor der Herausforderung mehr als bisher, theoretische Bezugsrahmen zu entwickeln und empirisch zu überprüfen, die das Verständnis bezüglich der Gestaltung digitaler Assistenzsysteme in der Kom- missionierung und der Rahmenbedingungen ihres Einsatzes (z.B. Aufgabentyp, Aufgabenträgermerkmale) so verbessern, dass es der Unternehmenspraxis leichter fällt, mit solchen Systemen angestrebte Leistungssteigerungen auch tatsächlich zu erreichen.

Funding Open Access funding provided by Projekt DEAL.

\section{Anhang A}

Tab. 5 Fragen der Skala „Empfundene Anforderungen“a Table 5 Questions underlying the "perceived workload" scale

- Wie hoch waren die geistigen Anforderungen (z. B. Koordination, Konzentration, Kommunikation), die dieses Assistenzsystem an Sie gestellt hat?

- Wie viel körperliche Anstrengung war nötig, um dieses Assistenzsystem zu bedienen?

- Wie stark mussten Sie sich insgesamt anstrengen, um den Kommissionierungsauftrag abzuarbeiten?

- Wie stark fühlten Sie sich bei der Kommissionierung überfordert (Stressempfinden)?

${ }^{a}$ Kursive Hervorhebungen entsprechen der Darstellung im Fragebogen

Tab. 6 Items der Skala „Einfachheit der AS-Nutzung“

Table 6 Items underlying the "ease of assistance system use" scale

- Das Arbeiten mit dem System hat mir Spaß gemacht

- Die Handhabung/Bedienbarkeit des Systems ist nicht schwierig

- Die Auftragsinformationen sind gut zu lesen

- Das Aufsuchen des Lagerplatzes ist mit dem System einfach

- Die Überprüfung der entnommenen Ware ist mit dem System unkompliziert

- Die Arbeit mit dem System ist interessant 


\section{Anhang B}

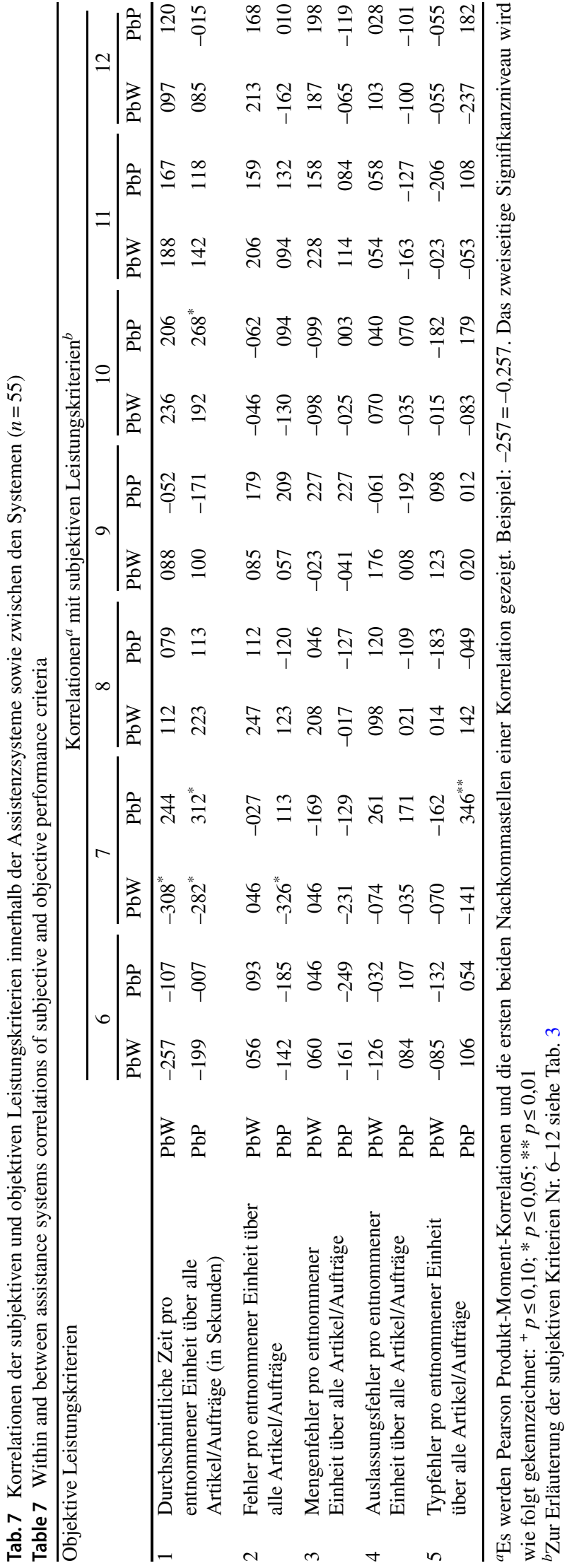

Open Access Dieser Artikel wird unter der Creative Commons Namensnennung 4.0 International Lizenz veröffentlicht, welche die Nutzung, Vervielfältigung, Bearbeitung, Verbreitung und Wiedergabe in jeglichem Medium und Format erlaubt, sofern Sie den/die ursprünglichen Autor(en) und die Quelle ordnungsgemäß nennen, einen Link zur Creative Commons Lizenz beifügen und angeben, ob Änderungen vorgenommen wurden.

Die in diesem Artikel enthaltenen Bilder und sonstiges Drittmaterial unterliegen ebenfalls der genannten Creative Commons Lizenz, sofern sich aus der Abbildungslegende nichts anderes ergibt. Sofern das betreffende Material nicht unter der genannten Creative Commons Lizenz steht und die betreffende Handlung nicht nach gesetzlichen Vorschriften erlaubt ist, ist für die oben aufgeführten Weiterverwendungen des Materials die Einwilligung des jeweiligen Rechteinhabers einzuholen.

Weitere Details zur Lizenz entnehmen Sie bitte der Lizenzinformation auf http://creativecommons.org/licenses/by/4.0/deed.de.

\section{Literatur}

Aromaa S, Aaltonen I, Kaasinen E, Elo J, Parkkinen I (2016) Use of wearable and augmented reality technologies in industrial maintenance work. In: Proceedings of the 20th International Academic Mindtrek Conference. ACM, Tampere, S 235-242

Bächler A (2017) Entwicklung und Evaluierung eines nutzerzentrierten Assistenzsystems zur Unterstützung von leistungsgeminderten Mitarbeitern bei manuellen Kommissioniertätigkeiten. Technische Universität Ilmenau, Ilmenau (Dissertation)

Baumann H (2013) Order picking supported by mobile computing. Universität Bremen, Bremen (Dissertation)

Beh PK, Ganesan Y, Iranmanesh M, Foroughi B (2019) Using smartwatches for fitness and health monitoring: The UTAUT2 combined with threat appraisal as moderators. Behav Inf Technol. https:// doi.org/10.1080/0144929X.2019.1685597

Bitkom (2019) Smartphone-Markt: Konjunktur und Trends. Bitkom, Berlin. https://www.bitkom.org/sites/default/files/2019-02/ Bitkom-Pressekonferenz\%20Smartphone-Markt\%2020\%2002 \%202019\%20Pr\%C3\%A4sentation_final.pdf. Zugegriffen: 19. Juni 2020

Blake C (2013) Eye-Tracking: Grundlagen und Anwendungsfelder. In: Möhring W, Schlütz D (Hrsg) Handbuch standardisierte Erhebungsverfahren in der Kommunikationswissenschaft. Springer, Berlin, S 367-387

Bröring A, Fast A, Büttner S, Heinz M, Röcker C (2019) Smartwatches zur Unterstützung von Produktionsmitarbeitern. In: Hess S, Fischer H (Hrsg) Mensch und Computer 2019. Gesellschaft für Informatik, Hamburg, S 228-235

Dehghani M, Kim KJ (2019) The effects of design, size, and uniqueness of smartwatches: perspectives from current versus potential users. Behav Inf Technol 38:1143-1153

Franzke T (2018) Der Mensch als Faktor in der manuellen Kommissionierung: Eine simulationsbasierte Analyse der Effizienz in Person-zur-Ware-Kommissioniersystemen. Springer Gabler, Wiesbaden

Funk M, Shirazi AS, Mayer S, Lischke L, Schmidt A (2015) Pick from here!-An interactive mobile cart using in-situ projection for order picking. In: Proceedings of the 2015 ACM International Joint Conference on Pervasive and Ubiquitous Computing. ACM, Osaka, S 601-609

Funk M, Mayer S, Nistor M, Schmidt A (2016) Mobile in-situ pickby-vision: order picking support using a projector helmet. In: Proceedings of the 9th ACM International Conference on Pervasive 
Technologies Related to Assistive Environments. ACM, Corfu, S 45-48

Funk M, Hartwig M, Backhaus N, Knittel M, Deuse J (2018) Nutzerevaluation von Assistenzsystemen für die industrielle Montage. In: Weidner R, Karafillidis A (Hrsg) Proceedings der dritten transdisziplinären Konferenz. Technische Unterstützungssysteme, die die Menschen wirklich wollen. Helmut-Schmidt-Universität, Hamburg, S 213-221

Funke C (2020) Pick-by-Vision - Die Brille für die Intralogistik. In: Voß PH (Hrsg) Logistik - die unterschätzte Zukunftsindustrie. Springer Gabler, Wiesbaden, S 107-114

Gerpott TJ, Kurt A (2020) Assistenzsysteme in der Kommissionierung. Wirtschaftswiss Stud 49(6): 17-23

Günthner WA, Rammelmeier T (2012) Auf dem Weg zur Null-FehlerKommissionierung. Neu entwickeltes Konzept mit Zukunftspotenzial. f $+\mathrm{h}$ Projektguide Intralogistik 2012 (Sonderausgabe), S $16-18$

Günthner WA, Blomeyer N, Reif R, Schedlbauer M (2009) Pickby-Vision: Augmented Reality unterstützte Kommissionierung. Forschungsbericht Technische Universität München. https:// mediatum.ub.tum.de/doc/1188222/file.pdf. Zugegriffen: 19. Juni 2020

Guo A, Raghu S, Xie X, Ismail S, Luo X, Simoneau J, Gilliland S, Baumann H, Southern C, Starner T (2014) A comparison of order picking assisted by head-up display (HUD), cart-mounted display (CMD), light, and paper pick list. In: Proceedings of the 2014 ACM International Symposium on Wearable Computers. ACM, Seattle, S 71-78

Händlerbund (2018) Logistik-Studie 2018, Teil 2: Lager \& Verpackung. Händlerbund, Leipzig. https://www.haendlerbund.de/de/ downloads/logistik-studie-teil-2-verpackung.pdf. Zugegriffen: 19. Juni 2020

Hara K, Umezawa T, Osawa N (2015) Effect of button size and location when pointing with index finger on smartwatch. In: Kurosu M (Hrsg) Human computer interaction: interaction technologies, part II. Springer, Heidelberg, S 165-174

ten Hompel M, Sadowsky V, Mühlenbrock S (2019) Kommissioniersysteme. In: Schmidt T (Hrsg) Innerbetriebliche Logistik. Springer Vieweg, Berlin, S 113-152

ten Hompel M, Schmidt T, Dregger J (2018) Materialflusssysteme: Förder- und Lagertechnik, 4. Aufl. Springer, Berlin

Initiative D21 (2019) D21 DIGITAL INDEX 2018/2019. https:// initiatived21.de/app/uploads/20s19/01/d21_index2018_2019.pdf. Zugegriffen: 19. Juni 2020

Kasselmann S, Willeke S (2016) Technologie-Kompendium: Interaktive Assistenzsysteme. IPH, Hannover. https://www.iph-hannover. de/_media/files/downloads/Projekt_40-Ready_Technologie-Kom pendium.pdf. Zugegriffen: 19. Juni 2020

Maienschein B (2017) Pick by Watch: Vom Handgelenk aus kommissionieren. https://www.mm-logistik.vogel.de/vom-handgelenkaus-kommissionieren-a-71132/. Zugegriffen: 19. Juni 2020

Murauer CS (2019) Full shift usage of smart glasses in order picking processes considering a methodical approach of continuous user involvement. Technische Universität Berlin, Berlin (Dissertation)

Niemöller C, Zobel B, Berkemeier L, Metzger D, Werning S, Adelmeyer T, Ickerott I, Thomas O (2017) Sind Smart Glasses die Zukunft der Digitalisierung von Arbeitsprozessen? Explorative Fallstudien zukünftiger Einsatzszenarien in der Logistik. In: Leimeister JM, Brenner W (Hrsg) Proceedings der 13. Internationalen Tagung Wirtschaftsinformatik WI 2017. Universität St. Gallen, St. Gallen, S 410-424
Park E (2020) User acceptance of smart wearable devices: an expectation-confirmation model approach. Telemat Inform 47:101318

Pfeiffer S, Lee HS, Zirnig C, Suphan A (2016) Industrie 4.0: Qualifizierung 2025. VDMA, Frankfurt/M.

Poller J, Wigger C, Lorson PC, Haustein E (2016) Kommunale Nachhaltigkeitsberichterstattung: Was interessiert Bürger als Hauptadressaten? ZögU 38:301-334

Rammelmeier T (2017) Vermeidung und prozessintegrierte Erkennung von Kommissionierfehlern auf Basis der Pick-by-Vision-Technologie. Technische Universität München, München (Dissertation)

Reichel J (2016) SSI Schäfer: Pick by Watch für mehr Effizienz. Mit einer Smart-Watch am Handgelenk soll das Person-zu-Ware-Kommissionieren noch schneller von statten gehen. http:// www.logistra.de/news-nachrichten/nfz-fuhrpark-lagerlogistikintralogistik/7178/test-amp-technik/ssi-schaefer-pick-watchfuer-mehr-effizienz. Zugegriffen: 19. Juni 2020

Reif R, Walch D (2008) Augmented \& virtual reality applications in the field of logistics. TVC 24:987-994

Schlick C, Bruder R, Luczak H (2018) Arbeitswissenschaft, 4. Aufl. Springer Vieweg, Berlin

Schwerdtfeger B (2010) Pick-by-vision: Bringing HMD-based augmented reality into the warehouse. Technische Universität München, München (Dissertation)

Statistisches Bundesamt (2019) Statistisches Jahrbuch Deutschland und Internationales 2019. https://www.destatis.de/DE/Themen/ Querschnitt/Jahrbuch/statistisches-jahrbuch-2019-dl.pdf?_blob= publicationFile. Zugegriffen: 19. Juni 2020

Stockinger C, Steinebach T, Petrat D, Bruns R, Zöller I (2020) The effect of pick-by-light-systems on situation awareness in order picking activities. Procedia Manuf 45:96-101

Stoltz MH, Giannikas V, McFarlane D, Strachan J, Um J, Srinivasan R (2017) Augmented reality in warehouse operations: opportunities and barriers. IFAC-Papers-OnLine 50:12979-12984

Thomas C, Panagiotopoulos T, Kotipalli P, Haynes M, Starner T (2018) RF-pick: Comparing order picking using a HUD with wearable RFID verification to traditional pick methods. In: Proceedings of the 2018 ACM International Symposium on Wearable Computers. ACM, Singapore, S 168-175

Winkler H, Zinsmeister L (2019) Trends in digitalization of intralogistics and the critical success factors of its implementation. Braz $\mathrm{J}$ Oper Prod Manag 16:537-549

Wu X, Haynes M, Zhang Y, Jiang Z, Shen Z, Guo A, Starner T, Gilliland $S$ (2015) Comparing order picking assisted by head-up display versus pick-by-light with explicit pick confirmation. In: Proceedings of the 2015 ACM International Symposium on Wearable Computers. ACM, Osaka, S 133-136

Wu X, Haynes M, Guo A, Starner T (2016) A comparison of order picking methods augmented with weight checking error detection. In: Proceedings of the 2016 ACM International Symposium on Wearable Computers. ACM, Heidelberg, S 144-147

Zerbe S (2019) Renaturierung von Ökosystemen im Spannungsfeld von Mensch und Umwelt. Springer Spektrum, Berlin

Univ.-Prof. Dr. Torsten J. Gerpott Inhaber des Lehrstuhls Unternehmens- und Technologieplanung, Schwerpunkt Telekommunikationswirtschaft, Mercator School of Management, Universität DuisburgEssen

Ahmet Kurt Mitarbeiter am Lehrstuhl Unternehmens- und Technologieplanung, Schwerpunkt Telekommunikationswirtschaft, Mercator School of Management 